Review Article

\title{
LIQUISOLID TECHNIQUE: A NOVEL APPROACH FOR DOSAGE FORM DESIGN
}

\author{
SATYAJIT PANDA ${ }^{*}$, R. VARAPRASAD ${ }^{2}$, K. PRIYANKA ${ }^{2}$, RANJIT P. SWAIN ${ }^{2}$
}

1Department of Pharmaceutics, Institute of Pharmacy and Technology, Salipur, Cuttack (Odisha) 754202, India, ${ }^{2}$ Department of Pharmaceutical Technology, Maharajah's College of Pharmacy, Phool Baugh, Vizianagram (A. P.) 535002, India

Email: satya.jcp@gmail.com

Received: 25 Mar 2017, Revised and Accepted: 17 Apr 2017

\section{ABSTRACT}

The liquisolid technique is a novel approach for delivery of drugs through the oral route. This technique is suitable for poorly soluble or water insoluble drugs, highly permeable drugs (BCS Class II drugs) and also for immediate or sustained release formulations. It is a novel "Powder Solution Technology" that involves absorption and adsorption efficiencies, making use of liquid medications, drug suspensions admixed with suitable carriers, coating materials and formulated into free flowing, dry looking, non-adherent and compressible powder forms. The design of liquisolid systems are mainly intended for enhancement of solubility, dissolution rate and bioavailability of poorly water-soluble and highly lipophilic drugs. Improvement in bioavailability may be due to increased surface area, increased aqueous solubility and increased the wettability of the drug. Liquisolid technique also has the potential to be optimized for the reduction of drug dissolution rate and thereby production of sustained release systems. Overall, liquisolid technique is a most promising and novel technique for enhancing the dissolution and bioavailability of poorly water soluble drugs and sustaining drug release from tablet matrices. The current review mainly focuses on theory and applicability of liquisolid compact technique towards solubility or bioavailability enhancement. Different carriers, solvents and coating materials employed are elucidated. Literature reports on the applicability of liquisolid compact techniques over a wide range of pharmaceutical formulations are also explicated.

Keywords: Lipophilic, Bioavailability, Wettability, Carrier, Sustaining

(c) 2017 The Authors. Published by Innovare Academic Sciences Pvt Ltd. This is an open access article under the CC BY license (http://creativecommons.org/licenses/by/4.0/) DOI: http://dx.doi.org/10.22159/ijap.2017v9i3.18698

\section{INTRODUCTION}

Out of the numerous challenges in the design of pharmaceutical dosage forms, the most important is the solubility enhancement of poorly water-soluble drugs and improvement of bioavailability [1].
In recent years, the number of drug candidate has increased. However, most of these drugs are highly lipophilic and poorly water-soluble about $40 \%$ of the newly developed drugs and nearly $60 \%$ of the synthesised chemical entities suffer from solubility issues $[2,3]$

Table 1: Descriptive terms for solubility according to Indian pharmacopoeia [4]

\begin{tabular}{lll}
\hline S. No. & Descriptive terms & Parts of solvent required to dissolve one part of solute (m) \\
\hline 1 & Very soluble & Less than 1 \\
2 & Freely soluble & More than 1 but less than 10 \\
3 & Soluble & More than 10 but less than 30 \\
4 & Sparingly soluble & More than 30 but less than 100 \\
5 & Slightly soluble & More than 100 but less than 1000 \\
6 & Very slightly soluble & More than 1000 but less than 10,000 \\
7 & Insoluble & More than 10,000 \\
\hline
\end{tabular}

Those belonging to the BCS class II and IV, dissolve poorly, slowly, and irregularly and hence possess serious delivery challenges like the incomplete release of drug from the dosage form, poor bioavailability of drug and high inter-patient variability [5].

Table 2: Biopharmaceutical classification system [5]

\begin{tabular}{|c|c|c|c|c|}
\hline S. No. & BCS class & Solubility & Permeability & Examples \\
\hline 1 & I & High & High & Metoprolol, Diltiazem, Verapamil, Propranolol, \\
\hline 2 & II & Low & High & Danazol, Nifedipine, Ketoprofen, Naproxen, \\
\hline 3 & III & High & Low & Atenolol, Captopril Ranitidine, Acyclovir \\
\hline 4 & IV & Low & Low & Taxol, Furosemide. \\
\hline
\end{tabular}

\section{Techniques for solubility enhancement}

Many approaches have been developed for enhancement of solubility of poorly water-soluble and lipohilic drugs. Micronization technique is the most commonly used approach to improve drug solubility of poorly soluble drugs due to an increase in surface area. Other approaches, such as inclusion complexes, microencapsulation, and preparation of selfnanoemulsions and solid lipid nanoparticles have also been studied for dissolution enhancement of poorly water-soluble drugs [2].

\section{Liquisolid compact technique}

The liquisolid technique is a novel and most promising technique for improving the dissolution rate of poorly water-soluble drugs. In this technique with the use of carrier and coating materials the liquid form of drug converted into dry looking, non-adherent, free flowing, and directly compressible powder. In liquisolid system, the liquid portion is a drug suspension, liquid drug, or drug solution made in suitable non-volatile liquid vehicles $[6,7]$. 


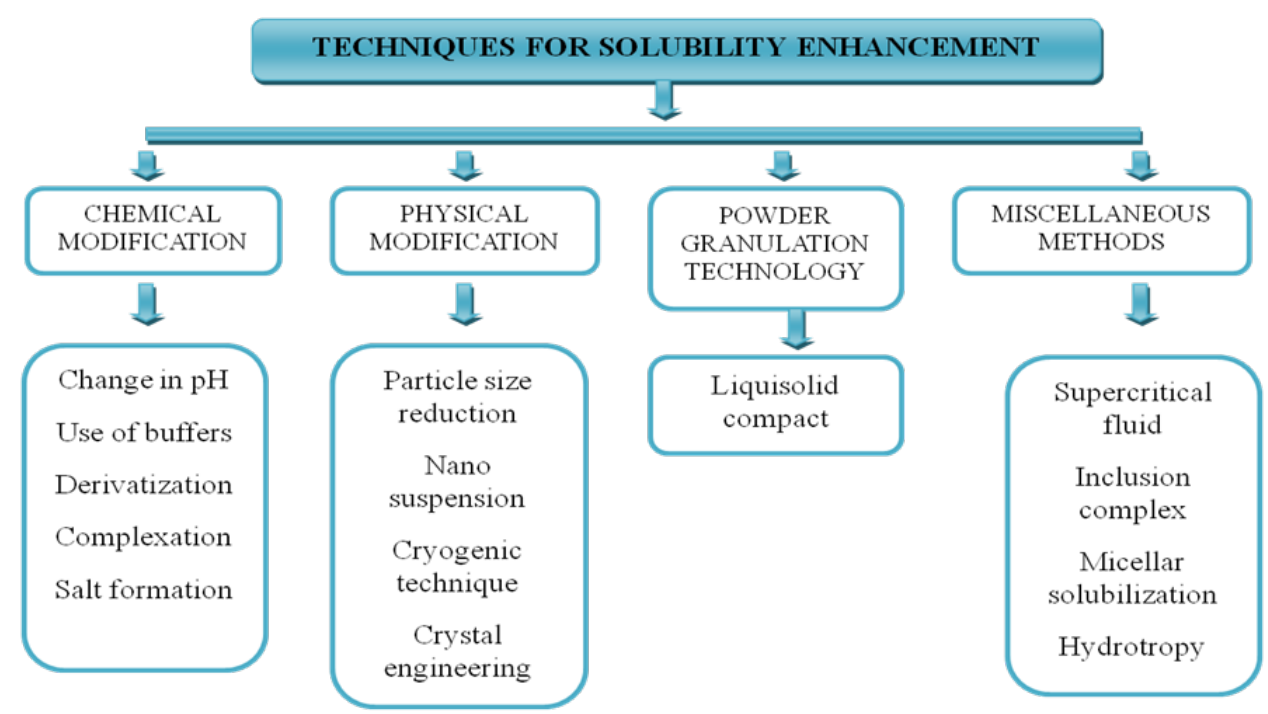

Fig. 1: Solubility enhancement techniques [2]

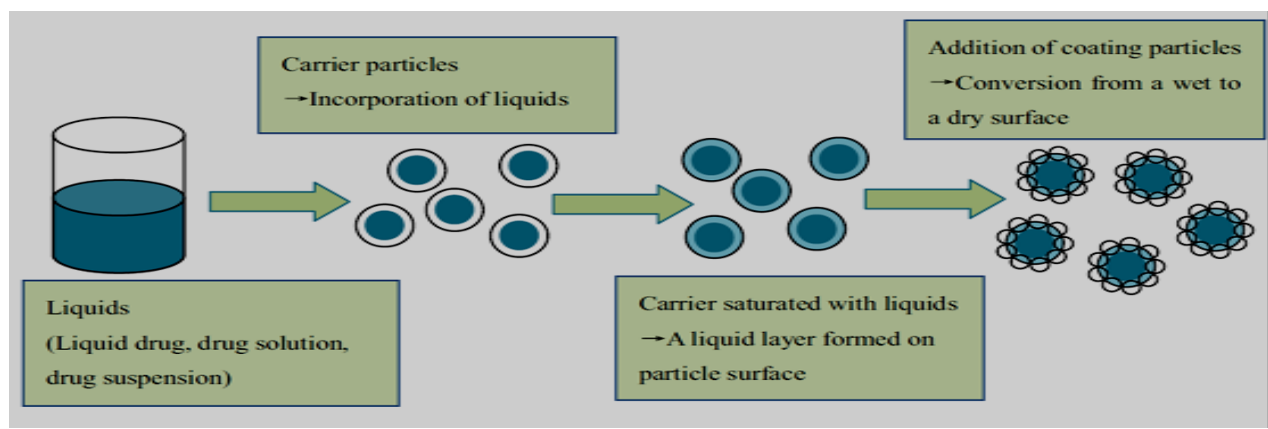

Fig. 2: Liquisolid compact formation $[6,7]$

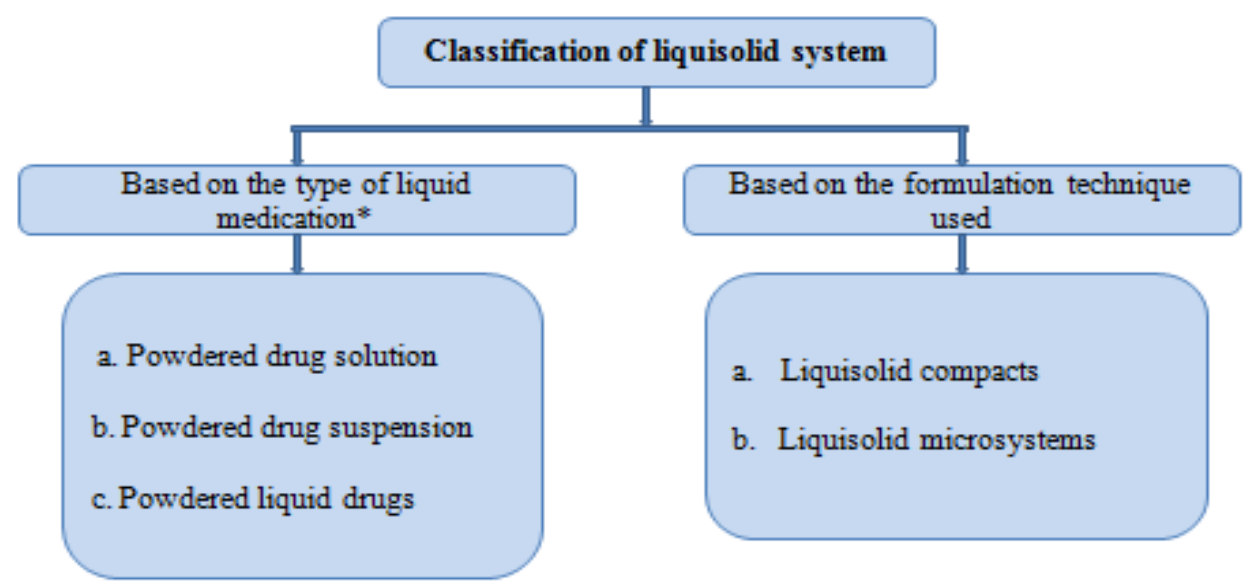

Fig. 3: Classification of liquisolid system $[7,8]$

*Powdered drug solution, suspension, the liquid drug is produced from the conversion of drug solutions or drug suspensions, and formulation of liquid drugs into liquisolid systems.

\section{Theory of liquisolid system [2, 9-11]}

For the production of liquisolid systems amounts of powder excipients required is calculated by using a mathematical approach developed by Spireas. This approach is depends on the flowable $(\varnothing$ value) and compressible ( $\varphi$-number) liquid retention potential introducing constants for each powder/liquid combination.
The $\varphi$-number of a powder is defined as the maximum amount of non-volatile liquid the powder can retain inside its bulk while maintaining acceptable compatibility resulting in compacts of sufficient hardness during compression. It can be measured as the maximum crushing strength of one-gram tablet compacted at sufficiently high compression forces.

The $\emptyset$-value of a powder is defined as the maximum amount of a given non-volatile liquid that can be retained inside its bulk while maintaining an acceptable flowability. 
The liquid load factor that ensures acceptable flowability $\left(\emptyset_{\mathrm{Lf}}\right)$ can be determined by:

$$
\mathrm{Lf}=\varnothing+\varphi(1 / \mathrm{R})
$$

Where, $\varnothing$ and $\varphi$ are the $\emptyset$-values of the carrier and coating material, respectively.

Depending on the excipient ratio $(\mathrm{R})$ of the powder substrate an acceptably flowing and compressible liquisolid system can be obtained only if a maximum liquid load on the carrier material is not exceeded.

$$
\mathrm{R}=\mathrm{Q} / \mathrm{q}
$$

$R$ represents the ratio between the weights of the carrier $(Q)$ and the coating (q) material present in the formulation.

By using the following equation we can measure the quantities of carriers $\left(Q_{0}\right)$ and coating $\left(q_{0}\right)$ materials are required to convert liquid formulation (W) into acceptably flowing and directly compressible powder.

$$
\begin{aligned}
& \mathrm{Q}_{0}=\mathrm{W} / \mathrm{L}_{0} \\
& \mathrm{q}_{0}=\mathrm{Q}_{0} / \mathrm{R}
\end{aligned}
$$

Table 3: $\varnothing$-values and $\varphi$-values of different carrier and coating materials [12]

\begin{tabular}{lllll}
\hline S. No. & Powder excipients & $\emptyset$ value & \multicolumn{1}{c}{$\boldsymbol{\varphi \text { value }}$} \\
\cline { 3 - 5 } & & Propylene glycol & PEG 400 & Propylene glycol \\
\hline 1 & Avicel PH102 & 0.16 & 0.005 & 0.224 \\
2 & Avicel PH200 & 0.26 & 0.02 & 0.209 \\
3 & Cab-O-Sil M5 with Avicel PH 102 & 3.31 & 3.26 & 0.560 \\
4 & Cab-O-Sil M5 with Avicel PH 200 & 2.56 & 2.44 & 0.232 \\
\hline
\end{tabular}

\section{Enhanced drug release mechanism from liquisolid systems}

Three main mechanisms are involved for enhancement of drug release from liquisolid systems are as follows

\section{Increased drug surface area}

In liquisolid system the surface area of drug available for drug release is much greater than that of drug particles within directly compressed tablets because the drug present in the liquisolid system is completely dissolved in the liquid vehicle and present in the powder substrate still in a solubilized, molecularly dispersed state [9].

Consequently, with increasing drug content, the solubility limit also increases and thus, increasing the fraction of undissolved drug in the liquid vehicle and thus, the release rate decreases. In the liquid solid formulation, the release rate of the drug is directly proportional to the fraction of the molecularly dispersed drug (FM). Spireas defined FM as the ratio of the drug solubility (Sd) and the actual drug concentration (Cd) in the liquid vehicle [13].

$$
\mathrm{FM}=\mathrm{Sd} / \mathrm{Cd}
$$

Where $\mathrm{FM}=1 \mathrm{Sd} \geq \mathrm{Cd}$
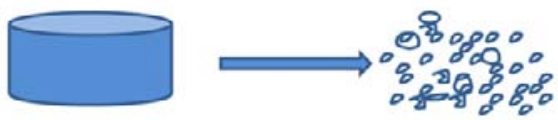

Fig. 4: Increased drug surface area [13]

\section{Increased aqueous solubility of the drug}

The solubility of the drug may be increased with liquisolid system. In fact, the small amount of the liquid vehicle in a liquisolid compact is not sufficient to increase the overall solubility of the drug in the aqueous dissolution medium. If the small amount of liquid vehicle acts as a co- solvent in liquisolid system this less amount of vehicle is sufficient to increase the aqueous solubility of the poorly water soluble drug [14].

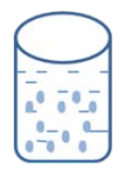

Dispersion

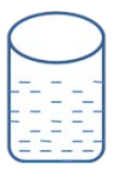

Clear solution
Fig. 5: Increased aqueous solubility of drug [14]

\section{Increased wettability}

The non-volatile solvent present in the liquisolid system provides wetting of drug particles by decreasing interfacial tension between tablet surface and dissolution medium so the contact angle of liquisolid system is lower when compared to the conventional formulation thus improved wettability [13].

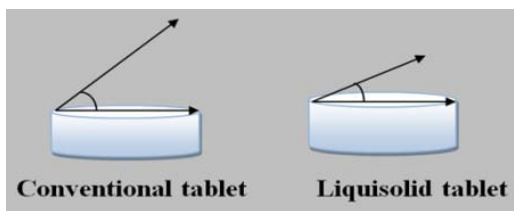

Fig. 6: Contact angle of conventional and liquisolid tablets [13]

\section{Requirements for preparation of liquisolid systems} Drug candidates [15]

Drug substance with solubilities below $0.1 \mathrm{mg} / \mathrm{ml}$ face significant solubilization obstacles, and often even compounds with solubilities below $10 \mathrm{mg} / \mathrm{ml}$ present difficulties related to solubilization during formulation.

Table 4: Drugs used in liquisolid systems [15]

\begin{tabular}{lll}
\hline S. No. & Name of the drug & Use \\
\hline 1 & Griseofulvin & Anti-fungal \\
2 & Lovastatin & Hypertriglyceridemia \\
3 & Felodipine & Anti-hypertension \\
4 & Budesonide & Anti-asthmatic \\
5 & Aceclofenac & NSAIDS \\
6 & Carbamazepine & Anti-epileptic \\
7 & Nevirapine & Anti-viral \\
8 & Praziquantel & Anti-helmeted \\
9 & Trimethoprim & Anti-biotic \\
10 & Clofibrate & Antihyperlipidemic \\
\hline
\end{tabular}




\section{Non-volatile solvent}

Non-volatile solvents used in the liquisolid systems should be safe, water-miscible, inert, not highly viscous. The carriers and coating materials required to prepare the liquisolid system decreases with increase in the solubility of the drug in a non-volatile solvent [16].

\section{Carrier materials}

Carriers used in liquisolid systems should have a porous surface and high liquid absorption capacity. Specific surface area and liquid absorption capacity are the most important properties of carriers and these carriers incorporate large amount liquid in its structure $[2,16]$.

\section{Coating materials}

The coating material in liquisolid system should be a material possessing fine (0.01-5 $\mu \mathrm{m}$ in diameter) and highly absorptive particles, which contribute to covering the wet carrier particles and displaying a dry powder by adsorbing excess amount of liquid to ensure good flowability of created blend [5].

Table 5: Various non-volatile solvents used in liquisolid system [6]

\begin{tabular}{lll}
\hline S. No. & Non-volatile solvent & HLB value \\
\hline 1 & Propylene glycol & 2.5 \\
2 & Polyethylene glycol 200 monostearate & 8 \\
3 & Polyethylene glycol 400 monostearate & 11.5 \\
4 & Polysorbate 80 & 15 \\
5 & Capryol $^{\text {TM } 90}$ & 5 \\
\hline
\end{tabular}

Table 6: various types of carrier materials used in liquisolid system [2, 6]

\begin{tabular}{lll}
\hline S. No. & Carrier material & Specific surface ${\operatorname{area~}\left(\mathbf{m}^{2} / \mathbf{g}\right)}$ \\
\hline 1 & Microcrystalline cellulose & 1.18 \\
2 & Lactose & 0.35 \\
3 & Sorbitol & 0.37 \\
4 & Starch & 0.6 \\
5 & Fujicalin ${ }^{\circledR}$ & 40 \\
6 & Neusilin ${ }^{\circledR}$ & 300 \\
\hline
\end{tabular}

Table 7: Various types of coating materials used in liquisolid system [6]

\begin{tabular}{llll}
\hline S. No. & Coating material & Composition & Specific surface area(m $\left.{ }^{2} / \mathbf{g}\right)$ \\
\hline 1 & Cab-O-Sil ${ }^{\circledR}$ M5-P & Untreated fumed silica \\
2 & Syloid $^{\circledR}$ & Amorphous silicon dioxide & 220 \\
3 & Aerosil $^{\circledR} 200$ & Hydrophilic fumed silica & 312 \\
4 & Neusilin $^{\circledR}$ & Amorphous aluminomagnesium metasilicate & 200 \\
\hline
\end{tabular}

\section{other additives}

The disintegration of solid dosage forms noticeably influences drug release, Sodium starch glycolate is most commonly used disintegrant in the formulation of liquisolid tablets [1].

Polyvinylpyrrolidone (PVP) is another promising additive, which has the potential to incorporate a high amount of drug into liquisolid systems and minimizes the overall tablet weight.

There is another additive used in liquisolid systems-HPMC, which usually acts as a release retarding agent to sustain drug release from liquisolid tablet [8].

\section{Advantages of liquisolid systems $[11,15,17]$}

1. Liquisolid technique has the potential to formulate liquisolid tablets or capsules with $\mathrm{pH}$-independent drug release pro files.

2. Enhanced bioavailability can be obtained in liquisolid technique.

3. Though the drug is in a tableted dosage form it is held in a solubilized liquid state, which increases drug wetting properties, drug dissolution rate and bioavailability.

4. Industrial production of liquisolid tablets or capsules is Possible.

5. Water insoluble drugs exhibit enhanced in vivo and in-vitro drug release profiles in the liquisolid system.

6. For the formulation of the liquisolid system, less excipients are required when compared to conventional formulations.

7. Increased surface area of drug exposed to dissolution medium.
8. By using hydrophobic carriers like Eudragit ${ }^{\circledR}$ RL and RS formulation of sustained release liquisolid tablets are possible.

9. Production cost is low when compared to soft gelatin capsules.

10. Liquisolid approach omits the process approaches like nanonisation, micronization techniques.

\section{Disadvantages of liquisolid systems [18-20]}

1. Liquisolid systems require high solubility of drug in nonvolatile solvents.

2. High levels of carrier material and coating materials should be required in order to achieve acceptable flowability and compactibility for liquisolid powder formulation.

Liquisolid system is the problematic formulation of a high dose of poorly water soluble drugs (e. g., carbamazepine, budesonide).

\section{Advantages of liquisolid systems $[11,15,17]$}

11. Liquisolid technique has the potential to formulate liquisolid tablets or capsules with pH-independent drug release profiles.

12. Enhanced bioavailability can be obtained in liquisolid technique.

13. Though the drug is in a tableted dosage form it is held in a solubilized liquid state, which increases drug wetting properties, drug dissolution rate and bioavailability.

14. Industrial production of liquisolid tablets or capsules is Possible.

15. Water insoluble drugs exhibit enhanced in vivo and in-vitro drug release profiles in liquisolid system. 
16. For the formulation of liquisolid system less excipients are required when compared to conventional formulations.

17. Increased surface area of drug exposed to dissolution medium.

18. By using hydrophobic carriers like Eudragit ${ }^{\circledR} \mathrm{RL}$ and $\mathrm{RS}$ formulation of sustained release liquisolid tablets are possible.

19. Production cost is low when compared to soft gelatin capsules.

20. Liquisolid approach omits the process approaches like nanonisation, micronization techniques.

\section{Disadvantages of liquisolid systems [18-20]}

3. Liquisolid systems require high solubility of the drug in nonvolatile solvents.

4. High levels of carrier material and coating materials should be required in order to achieve acceptable flowability and compatibility for liquisolid powder formulation.

5. Liquisolid system is the problematic formulation of a high dose of poorly water soluble drugs (e. g., carbamazepine, budesonide).

\section{Applications of liquisolid systems [21-25]}

1. Drug photostability in solid dosage forms is improved by liquisolid technique.
2. The rapid and prolonged release of drugs is obtained in liquisolid formulations.

3. The liquisolid technique is most efficiently used for waterinsoluble solid drugs or liquid lipophilic drugs.

4. Liquisolid technique minimizes effect of $\mathrm{pH}$ variation on drug release.

Drug solubility, dissolution rate is enhanced in liquisolid technique.

\section{Recent reports on liquisolid technique}

Mustafa E et al. (2017) had designed orodispersible tablets of zolmitriptan by using liquisolid technique. Orodispersible tablets were prepared by using propylene glycol, avicel PH-102 and aerosil 200 as a non-volatile solvent, carrier material and coating material respectively and various types of super disintegrating agents such as croscarmellose sodium, sodium starch glycolate, and crospovidone to facilitate faster disintegration of the liquisolid compact.

The overall results showed that among the three superdisintegrants, crospovidone was the best super disintegrant showing the shortest disintegration time while loading factor of 0.125 was the best in the preparing of zolmitriptan liquidsolid orodispersible tablets [26].

\section{Procedure for designing of liquisolid formulation}

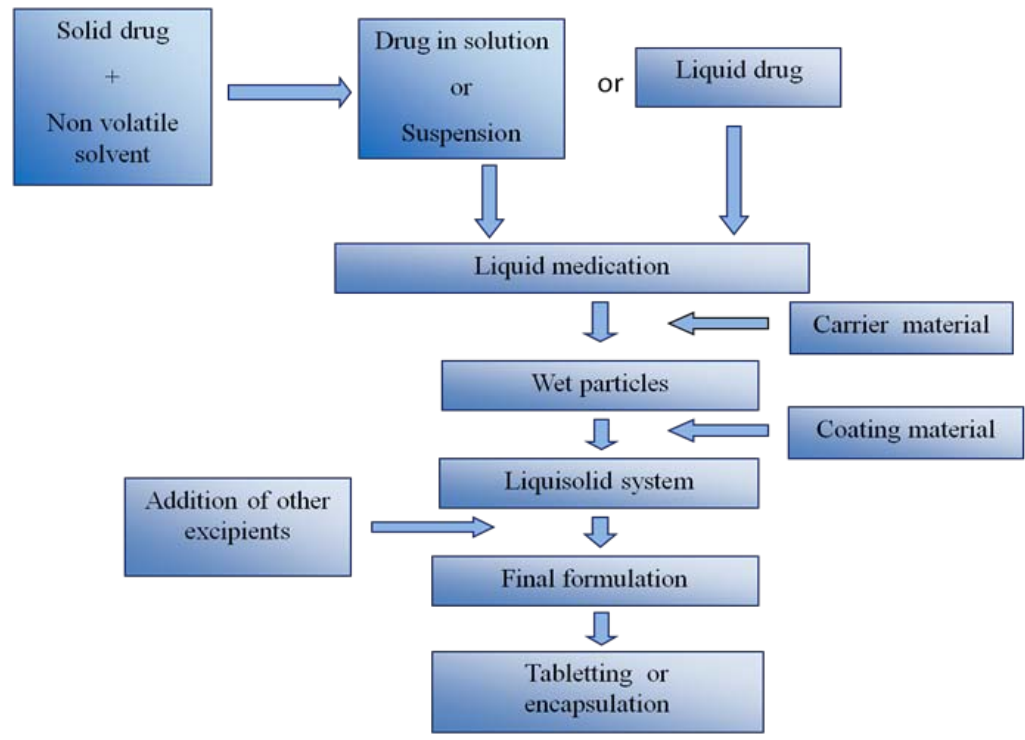

Fig. 7: General preparation procedure of liquisolid formulation [2]

Padmapreetha J et al. (2016) had formulated liquisolid compact to enhance the dissolution rate of leflunomide by using kolliphor EL, avicel PH 102, aerosol, and sodium starch glycolate as a non-volatile solvent, carrier, coating material and super disintegrant respectively. The results showed that during the first $10 \mathrm{~min}\left(\mathrm{Q}_{10 \%}\right)$ the optimized formulation released $73.39 \%$ of its content compared to $18.94 \%$ of the conventional formulation. In conclusion, leflunomide dissolution rate can be enhancing to a greater extent by liquisolid technique [27].

Mowafaq MG et al. (2015) had prepared liquisolid compact for solubility enhancement of tenoxicam using tween 80 as a nonvolatile liquid, avicel PH102 as a carrier, and aerosil 200 as a coating material. Liquisolid formulations containing various drug concentrations in liquid medication ranging from $10 \%$ to $35 \% \mathrm{w} / \mathrm{w}$ were prepared. Liquisolid formulations showed greater drug release rates than conventional and marketed tablets due to increasing surface area of the drug and wetting properties [28].
Hitesh J et al. (2014) had compared liquisolid and inclusion complexation techniques for dissolution rate enhancement of valsartan. This study was designed for screening of suitable nonvolatile liquid solvent for the preparation of liquisolid compact such as tween 80 , polyethyleneglycol 400 and propylene glycol by using the mathematical equation. The study was also aimed for enhancement of dissolution rate and comparison of liquisolid technique with inclusion complex of $\beta$-cyclodextrin. The liquisolid formulation showed highest dissolution rate compared with directly compressed tablet, pure drug, and formulation prepared by complexation technique [29].

Yesubabu B et al. (2014) had formulated fast disintegrating tablets of lamotrigine using different super disintegrating agents such as crospovidone, sodium starch glycolate. Various batches of liquisolid tablets were prepared. Formulations consisting of sodium starch glycolate were found to be fulfilling all the parameters satisfactorily 
when compared with crospovidone. In-vitro, drug release studies showed that within 30 min almost $90 \%$ of the drug was released from all the formulations confirming enhancement of drug dissolution by liquisolid technique [30].

Ali $\mathrm{N}$ et al. (2008) had designed sustained release liquisolid compact of propranolol hydrochloride by dispersing the drug in polysorbate 80 as the non-volatile solvent. A binary mixture of Eudragit RL or RS were used as the carrier. Silica was added to the liquid medium as the coating material with continuous mixing in a mortar. Then the final mixture was compressed using the tablet punching machine.

The effect of drug concentration, loading factor, thermal treating and aging on drug release profiles of propranolol $\mathrm{HCl}$ from liquisolid compacts was investigated at two different $\mathrm{pH}$ values such as 1.2 and 6.8. In conclusion, propranolol $\mathrm{HCl}$ tablets prepared by liquisolid system showed greater retardation properties than conventional matrix tablets [31].

Table 8: Literature reports on formulations of liquisolid compact

\begin{tabular}{|c|c|c|c|c|c|}
\hline $\begin{array}{l}\text { S. } \\
\text { No. }\end{array}$ & Drug & Non-volatile solvent & Carrier material & $\begin{array}{l}\text { Coating } \\
\text { material }\end{array}$ & $\begin{array}{l}\text { Ref. } \\
\text { No. }\end{array}$ \\
\hline 1 & Budesonide & PEG 400 & Avicel PH 102 & Aerosil 200 & 19 \\
\hline 2 & Carvedilol & PEG 400 & Avicel PH 101 & Aerosil 200 & 32 \\
\hline 3 & Candesartan cilexetil & Tween 80 & Avicel PH 102 & Aerosil 200 & 33 \\
\hline 4 & Efavirenz & Propylene glycol & Avicel PH 102 & Aerosil 200 & 34 \\
\hline 5 & Felodipine & PEG 400 & Avicel PH 102 & Aerosil 200 & 35 \\
\hline 6 & Gliclazide & Acrysol EL 135 & Avicel PH 102 and Neusilin & Aerosil 200 & 36 \\
\hline 7 & Glyburide & PEG 400 & Avicel PH 101 and 102 & Aerosil 200 & 37 \\
\hline 8 & Hydrochlorothiazide & PEG 400+Water+Tween 60 & Microcrystalline cellulose & $\begin{array}{l}\text { Colloidal silicon } \\
\text { dioxide }\end{array}$ & 38 \\
\hline 9 & Ibuprofen & PEG 400 & Avicel PH 101 & Aerosil & 39 \\
\hline 10 & Indomethacin & Glycerin & Micro crystalline cellulose & Silica & 40 \\
\hline 11 & Naproxen & $\begin{array}{l}\text { PEG 400, 200, 600, PG, Glycerin, Tween 80, } \\
\text { Cremophor EL and Poloxamer } 181\end{array}$ & $\begin{array}{l}\text { Microcrystalline cellulose and } \\
\text { Dicalcium phosphate }\end{array}$ & Colloidal silica & 12 \\
\hline 12 & Nateglinide & PEG 400 & Avicel PH 102 & Aerosil 200 & 41 \\
\hline 13 & Nifedipine & $\begin{array}{l}\text { PEG 400, PG and } \\
\text { Tween } 80\end{array}$ & Avicel PH 102 & $\begin{array}{l}\text { Silica gel } \\
\text { powder }\end{array}$ & 42 \\
\hline 14 & Olmesartan medoxomil & Acrysol EL 135 & $\begin{array}{l}\text { Avicel PH102, Fujicalin and } \\
\text { Neusilin }\end{array}$ & Aerosil & 43 \\
\hline 15 & $\begin{array}{l}\text { Trimetazidine } \\
\text { dihydrochloride }\end{array}$ & Tween 80 & Avicel PH 102 & Aerosil & 44 \\
\hline 16 & Tramadol hydrochloride & Propylene glycol & Avicel PH 102 & Aerosil 200 & 45 \\
\hline
\end{tabular}

\section{CONCLUSION}

Enhancement of the solubility and dissolution rate of poorly watersoluble drugs is still a major challenge for pharmaceutical scientists. At the same time sustaining the drug release from dosage forms helps in a better and proper utilization of the drug. Both of these applications are major requisites for enhancement of drug bioavailability. Finally, from this review, it can be concluded that, among the various techniques involved for the drug bioavailability enhancement, liquisolid technology is one of the most promising approaches. It is found to be a multipotential and promising technology for dosage form development, because of the process simplicity, low economic inputs during production and possible industrial feasibility due to the good flow and compaction characteristics of liquisolid formulations.

\section{ACKNOWLEDGMENT}

The authors are expressing sincere thanks to the principal and management of Institute of Pharmacy and Technology, Salipur, Cuttack, Odisha and M. R. College of Pharmacy, Vizianagaram, A. P. for supporting the work.

\section{CONFLICTS OF INTERESTS}

Declare none

\section{REFERENCES}

1. Vimalson DC, Parimalakrishnan S, Jeganathan NS, Anbazhagan S. Techniques to enhance the solubility of hydrophobic drugs: an overview. Asian J Pharm Sci 2016;10:67-75.

2. Pingtian D, Dongkai $\mathrm{W}$, Mei L, Haonan X, Jingzheng J, Xiao C, et al. Liquisolid technique and its applications in pharmaceutics. Asian J Pharm Sci 2016;9:1-9.

3. Rajeshbabu V, Areefulla SH, Mallikarjun V. Solubility and dissolution enhancement: an overview. J Pharm Res 2010;3:141-5.
4. Kadam SV, Shinar DM, Saudagar RB. Review on solubility enhancement techniques. Int J Pharm Biol Sci 2013;3:462-75.

5. Srujan Kumar M, Balaji A, Kanakaiah K, Mahalakshmi K. Liquisolid compacts: a novel approach for the enhancement of solubility of poorly soluble drugs. Indo Am J Pharm Res 2013;3:4386-95.

6. Anjan KM, Sastry GM, Ranjit PS, Murthy PN, Narahari NP. Liquisolid technique: a novel approach in pharmaceutical formulation development. J Chem Pharm Res 2014;6:529-43.

7. Shashidher B, Madhusudhanrao Y, Venkateswarlu V. The liquisolid technique: an overview. Braz J Pharm Sci 2011;47:475-82.

8. Kulkarni AS, Nagesh HA, Madhav SM, Jayashree BG. Liquisolid system: a review. Int J Pharm Sci Nanotechnol 2010;3:795-802.

9. Manpreet K, Rajni B, Sandeep A. Liquisolid technology: a review. Int J Adv Pharm Sci 2013;4:1-5.

10. Utsav SP, Khushbu CP. Liquisolid technique for poorly soluble drugs. J Sci Innovative Res 2013;2:145-59.

11. Doijad RC, Pathan AB, Gaikwad SS, Baraskar SS, Pawar NB, Maske VD. Liquisolid: a novel technique for dissolution enhancement of poorly soluble drugs. Int J Curr Pharm Res 2012;3:735-49.

12. Vijaykumar N, Sridhar T, Anilkumar C, Pragathi K. Enhancement of oral bioavilability of naproxen by liquisolid compaction technology: in vitro, in vivo evaluation. Indo Am J Pharm Res 2013;3:1359-69.

13. Bhumi BP, Chainesh NS. Recent research on liquisolid technology for solubility enhancement-a review. Int J Adv Pharm 2016;5:1-7.

14. Harshil MP, Chainesh NS. A review-recent research on liquisolid compact for solubility and dissolution enhancement. J Pharm Sci Biosci Res 2016;6:628-34.

15. Amrit BK, Indrajeet DG, Avinash HH, Pandurang ND, Satish BB. Liquisolid tablets: a novel approach for drug delivery. Int J Health Res 2009;2:45-50. 
16. Sandip V, Vishal S, Nilesh G, Bakde BV. Effect of dissolution rate by liquisolid compact approach: an overview. Int J Chem Pharm Sci 2012;1:1013-8.

17. Vijaykumar N, Ramarao T, Jayaveera KN. Liquisolid compacts: a novel approach to enhance bioavailability of poorly soluble drugs. Int J Pharm Biol Sci 2011;1:89-102.

18. Ganesh NS, Deecaraman, Vijayalakshmi KNS, Raju L, Ramesh B. Formulation and evaluation of sustained release lornoxicam by liquisolid technique. Int J Pharm Sci Rev Res 2011;11:53-7.

19. Surti N, Patel DS, Pipaliya RM. Liquisolid tablets for dissolution enhancement of a hypolipidemic drug. Indian J Pharm Sci 2015;77:290-8

20. Meena K, Sneha M. A review on pioneering technique-liquisolid compact and applications. Res J Pharm Biol Chem Sci 2015;6:220-7.

21. Lova Raju KNS, Ganesh NS, Ramesh B, Kavitha K. Effect of dissolution rate by liquisolid compact approach: an overview. Pharm Lett 2011;3:71-83.

22. Pradeepkumar $\mathrm{CH}$, Venugopalaiah $\mathrm{P}$, Praveen Kumar $\mathrm{CH}_{\text {, }}$ Gnanaprakash K, Gobinath M. liquisolid systems-an emerging strategy for solubilization and dissolution rate enhancement of bcs class-ii drugs. Int J Pharm Res Rev 2013;3:56-66.

23. Kishor SG, Sayyad FJ. liquisolid compact a review. Indian J Pharm Biol Res 2013;4:26-31.

24. Tushar NP, Zankhana PS, Bhagirath KP, Pritesh P. Formulation development and in vitro characterization of carvedilol sustain release tablet by using liquisolid technique. Pharmacophore 2014;5:451-66

25. Aher SS, Pagar PR, Saudagar RB. Formulation and evaluation of liquisolid compact tablet of budesonide. Int J Curr Res 2016;8:35093-102.

26. Mustafa E, Shaimaa NA. Design zolmitriptan liquisolid orodispersible tablets and their in vitro evaluation. Int J Pharm Pharm Sci 2017;9:297-303.

27. Padmapreetha J, Arulkumaran KSG. Effect of kolliphor El on dissolution rate of leflunomide liquisolid compacts. J Pharm Sci Res 2016;8:586-93.

28. Mowafaq MG, Kamil KA, Jaafar JI. Liquisolid compact as an approach for tenoxicam solubility enhancement using tween 80 as liquid vehicle. J Chem Pharm Res 2015;7:379-93.

29. Hitesh J, Pasha TY, Bais CS, Anil B. Comparision of liquisolid and inclusion complexation technique for dissolution rate enhancement of valsartan. Int Res J Pharm 2014;5:604-7.

30. Yesubabu B, Sunium MS, Koteswari P, Gopalakrishna CH, Ashok kumar Reddy D. Formulation design and development of fast disintegrating tablets of lamotrigine using liquisolid technique. Int J Pharm Res Scholars 2014;3:305-16

31. Ali N, Yousef J, Leila M. Liquisolid technique as a new approach to sustain propranolol hydrochloride release from tablet matrices. Int J Pharm 2008;6:102-8.
32. Sandhya P, Shadab K, Divya B, Rao KSKP, Subrahmanyam CVS. Formulation and evaluation of liquisolid compacts of carvedilol. IOSR J Pharm Biol Sci 2013;6:26-36.

33. Tulsankar SL, Sayyad FJ, Umesh BK. Design and development of liquisolid compact of candesartan cilexetil to enhance dissolution. J Pharm Res 2013;5:381-88.

34. Soujanya B, Srujankumar M, Subrahmanyam KV, Shubhrajit M. Enhancement of solubility of efavirenz by liquisolid compact technique. Int J Innov Pharm Sci Res 2013;1:347-59.

35. Bhushan A, Bhairav, Megha S, Jadhav RB, Saudagar. Formulation and evaluation of liquisolid tablet of felodipine. World J Pharm Sci 2016;5:1670-85.

36. Patel D, Anmol E, Jain H, Upadhyay U. Enhancement of dissolution rate of gliclazide by liquisolid technique. Scholars Acad J Pharm 2015:4:132-7.

37. Jyothi P, Mohiuddi MZ, Shankaraiah P, Saritha C, Venkatratnam D. Formulation and evaluation of glyburide liquisolid compacts. Int J Pharm Res Rev 2014;3:36-46.

38. Iqbal Z, Amjad K, Yasar S, Lateef A, Ismail, Zia U, et al. Enhancement of dissolution rate of class II drugs (Hydrochlorothiazide); a comparative study of the two novel approaches; solid dispersion and liquisolid techniques. Saudi Pharm J 2015;1:650-7.

39. Chuahan PV, Patel HK, Patel BA, Patel KN, Patel PA. Liquisolid technique for enhancement of dissolution rate of ibuprofen. Int J Pharm Res Scholars 2012;1:268-80.

40. Majid S, Jafar A, Katayoun MS, Reza EF, Shirin SR, Ala S. Enhancement of dissolution rate of indomethacin using liquisolid compacts Iran J Pharm Res 2011;10:25-34

41. Iizhar AS, Bhavani G. Liquisolid technique based tablets for enhancement of dissolution rate of nateglinide. Indo Am J Pharm Res 2014;4:2392-400

42. Tejaswi A, Padma P, Arun kumar N, Srujanreddy M. Enhancement of solubility of nifedipine by liquisolid compacts technique. Indo Am J Pharm Res 2013;3:3433-65.

43. Shailesh TP, Hitesh HB, Dashrath MP, Suresh KD, Chhaganbhai NP. Formulation and evaluation of liquisolid compacts for olmesartan medoxomil. J Drug Delivery 2013;10:1-9.

44. Syed IA, Pavani E, Noman S. Liquisolid technique based sustained release tablet of trimetazidine dihydrochloride. Drug Invent Today 2013;8:302-10.

45. Amrit BK, Indrajeet DG, Avinash HH, Pandurang ND. Evaluation of in vitro dissolution profile comparison methods of sustained release tramadol hydrochloride liquisolid compact formulations with marketed sustained release tablets. Drug Discovery Ther 2010;4:20-32.

\section{How to cite this article}

- $\quad$ Satyajit Panda, R Varaprasad, K Priyanka, Ranjit P Swain. Liquisolid technique: a novel approach for dosage form design Int J Appl Pharm 2017;9(3):8-14. 